\title{
Medizinethik und Empirie - Standortbestimmungen eines spannungsreichen Verhältnisses
}

\author{
Silke Schicktanz $\cdot$ Jan Schildmann
}

Online publiziert: 3. September 2009

(C) Die Autor(en) 2009. Dieser Artikel ist auf Springerlink.com mit Open Access verfügbar.

Die Empirische Ethik ${ }^{1}$ hat in der internationalen Fachdiskussion eine enorme Popularität erreicht. Dabei fällt mindestens zweierlei auf: Zum einen gibt es eine intensive Fachdebatte um theoretische, meta-ethische und methodologische Fragen, ob und wenn, auf welche Weise empirische Ergebnisse in medizinethische Überlegungen zu integrieren sind. Zum anderen ist die Anzahl von medizinethischen Studien, die explizit Umfragen oder Interviews mit Ärzten, Richtern, Pflegepersonal oder Patienten beinhalten, in den letzten Jahren enorm gestiegen. Die entsprechenden Arbeiten werden in den zwischenzeitlich von zahlreichen medizinethischen Fachzeitschriften unterhaltenen Rubriken zu empirical ethics oder clinical ethics publiziert. Die Verbindung von normativen, präskriptiven und empirischen, deskriptiven Dimensionen spiegelt sich u.a. darin wieder, dass angewandte Ethik zunehmend als ein interdisziplinäres Unternehmen verstanden wird. An diesem müssen nicht nur die Philosophie, Theologie und Rechtswissenschaft sowie die Medizin, die Natur- und Ingenieurswissenschaften mitwirken, sondern auch die Sozial- und Kulturwissenschaften.

Dieses Aktualitätsphänomen liefert aus unserer Sicht gute Gründe, sich mit dem Verhältnis von Medizinethik und Empirie näher auseinanderzusetzen. Die Verbindung normativer und empirischer Aspekte in der Medizinethik bietet enorme Chancen, aber auch Risiken, welche abzuschätzen und abzuwägen sind. In der aktuellen Entwicklung der Medizinethik spiegeln sich darin auch inhaltliche und strukturelle, institutionelle Veränderungen der ethischen Forschung in der Medizin wider. Aus inhaltlicher Sicht soll eine größere Praxisnähe

\footnotetext{
S. Schicktanz $(\square)$

Abteilung Ethik und Geschichte der Medizin, Universitätsmedizin Göttingen

Humboldtallee 36, 37073 Göttingen, Deutschland

E-Mail: silke.schicktanz@medizin.uni-goettingen.de

J. Schildmann

Institut für Medizinische Ethik und Geschichte der Medizin

Ruhr-Universität Bochum, Bochum, Deutschland

E-Mail: jan.schildmann@rub.de
}

\footnotetext{
${ }^{1}$ In der internationalen Fachliteratur sind hierfür verschiedene Begriffe zu finden wie: empirical ethics, empirical medical ethics, integrated empirical ethics oder empirically informed ethics. Wir verwenden hier der Einfachheit halber Empirische Ethik als Sammelbegriff.
} 
erzielt werden. Diese Praxisnähe wird sowohl bei der Problemerfassung, bei der Bewertung und als auch bei der Problemlösung eingefordert. Strukturell kann die stärkere empirische Ausrichtung der Medizinethik auch mit den Bedürfnissen der medizinischen Fakultäten in Verbindung gebracht werden, welche die Medizinethik am häufigsten beherbergen. Aber auch das verstärkte Interesse der Sozial- und Kulturwissenschaften an bioethischen Fragen dürfte kein unwesentlicher Faktor sein.

Allerdings sollte das Verhältnis zwischen den normativen Anteilen der Medizinethik einerseits und deskriptiver empirischer Forschung anderseits nicht als „harmlos“ eingestuft werden. Es wirft zahlreiche ungeklärte Fragen auf. Insbesondere interessiert die methodologische Spannung zwischen den ethischen und empirischen Dimensionen und was sie für die zukünftige Medizinethikforschung bedeuten könnte. Bislang fehlen gerade für die deutsche Fachdiskussion verschiedenartige Übersichten, die die methodischen Vor- und Nachteile der verschiedenen Ansätze in der Empirischen Ethik reflektieren. Auch praxisorientierte Handreichungen, die Restriktionen bestimmter Methoden hinsichtlich des Forschungsdesigns aufzeigen, sind eher noch die Ausnahme. Hierzu gehört eine an die sozialwissenschaftliche Diskussion heranreichende Methodenreflexion, welche Standards der empirischen Forschung einzuhalten und bei der Interpretation der Ergebnisse zu berücksichtigen sind. Schließlich dürfen die theoretischen Grundlagen nicht als hinreichend geklärt gelten. Bei genauerem Hinsehen wird deutlich, dass das, was unter Medizinethik beziehungsweise Empirie verstanden wird, immer noch stark variiert.

So wird Medizinethik im Sinne angewandter Ethik unter anderem a) als Reflexionstheorie, die die soziale Praxis reflektiert, b) als normative Reflexionstheorie, die den Anspruch hat, praktisch zu werden (Handlungsorientierung, Politikberatung), c) als Alltagsethik, d.h. als moralisches Nachdenken in konkreten Entscheidungen, wie in klinischen Situationen, oder d) als Prozess der Urteilsbildung, bei dem immer schon normative und ethisch-empirische Anteile verbunden werden müssen, verstanden.

Unterschiede mit Blick auf das Begriffsverständnis sind auch für die Empirie zu konstatieren. So wird unter Empirie a) das Sein bzw. „wissenschaftliche Fakten“, (im Gegensatz zum Sollen), das sich deskriptiv beschreiben lässt, b) die Alltagspraxis sowie c) die empirische Untersuchung moralischer Einstellungen und Meinungen verstanden.

Das vorliegende Themenheft „Medizinethik und Empirie“ hat sich nun die Aufgabe gemacht, die internationale Diskussion über Empirische Ethik für die deutsche Debatte aufzuarbeiten. In diesem Zusammenhang soll kritisch geprüft werden, was daran vielleicht ein „alter Hut" ist, das „Neue“ soll fruchtbringend für die Medizinethik herausgestellt werden.

In dem ersten einführenden Beitrag geht der holländische Philosoph Bert Musschenga genauer der Frage Was ist empirische Ethik? nach. Er stellt dabei mit Blick auf die internationale Debatte die spezifischen Kennzeichen einer Empirischen Ethik vor. Dabei zeigt er auf, dass die Diskussion um die methodischen Vorgehensweisen der angewandten Ethik wichtige Vorläufer hat. Anhand von verschiedenen Fallbeispielen aus der aktuellen Forschung werden eine ,generalistische-“ und „kontextuelle empirische Ethik“ voneinander abgegrenzt und jeweils die Stärken und Schwächen der unterschiedlichen Ansätze benannt.

In den folgenden drei Beiträgen wird das Verhältnis von Sein und Sollen in der Medizinethik aus der moralphilosophischen Perspektive stärker beleuchtet. In dem Aufsatz Wofür braucht die Medizinethik empirische Methoden? Eine normativ-ethische Untersuchung geht es Marcus Düwell um eine Analyse der meta-ethischen Herausforderungen im Verhältnis von Ethik und Sozialwissenschaften. Aus ethischer Perspektive kann nach Ansicht des Autors die Identifizierung wichtiger, sozial-empirischerer Forschungsdesiderate einen zentralen Beitrag zur medizinethischen Forschung leisten. Die mit der Durchführung entsprechender Forschung assoziierten methodologischen Herausforderungen sowie die hier- 
für erforderlichen methodischen Kompetenzen sind weitere Schwerpunkte der Arbeit. Mit Blick auf die ethische Urteilsbildung als ein Kerngeschäft der angewandten Ethik geht Julia Dietrich der Kraft der Konkretion oder: Die Rolle deskriptiver Annahmen für die Anwendung und Kontextsensitivität ethischer Theorie am Beispiel der Schmerztherapie nach. Sie analysiert die jeweils deskriptiven und normativen Prämissen im Rahmen eines konkreten medizinethischen Urteils. Dabei steht für sie die empirische Darstellung und Untersuchung als Prozess der Konkretisierung im Sinne der Bewegung von der allgemeinen zur angewandten Ethik im Zentrum der Überlegungen. Potentiale und Limitationen sozialempirischer Forschung für die Medizinethik aus normativer Sicht will auch Silke Schicktanz in ihrem Aufsatz Zum Stellenwert von Betroffenheit, Öffentlichkeit und Deliberation im „empirical turn“ der Medizinethik sichtbar machen. Ihr Schwerpunkt liegt auf der Frage, wessen Meinungen und Positionen vor allem mit sozialempirischen Untersuchungen eingeholt werden bzw. eingeholt werden sollen. Eine Chance sieht sie darin, die Perspektive „Betroffener“ sichtbarer als bisher zu machen. Allerdings sei das Ersetzen von Argumentation und Deliberation zugunsten von Meinungsumfragen auch ein Risiko des empirical turns. Sie argumentiert daher für eine kritischere Auseinandersetzung mit Konzeptionen von Betroffenheit, Öffentlichkeit und Expertise. Die normativen Annahmen, welche bei sozialempirischen Untersuchungen wichtig sind, müssen ihrer Ansicht nach stärker explizit gemacht und reflektiert werden.

Aus eher soziologisch geprägter Perspektive beschäftigen sich hingegen die nächsten zwei Beiträge mit dem Thema. Dem Verhältnis zwischen Medizinethik und Medizinsoziologie aus sozialwissenschaftlicher Perspektive gehen Sigrid Graumann und Gesa Lindemann in Medizin als gesellschaftliche Praxis, sozialwissenschaftliche Empirie und ethische Reflexion: ein Vorschlag für eine soziologisch aufgeklärte Medizinethik nach. Sie gehen vor allem von den eher strukturellen Bedingungen der Entwicklung der Medizinethik aus. Nach einem historischen Abriss zur Entwicklung der Medizinethik unter Berücksichtigung der Rolle verschiedener normativer und empirischer Disziplinen wird die stattgefundene Hinwendung der Medizinethik zur sozialwissenschaftlichen Forschung analysiert. Ihr Entwurf einer „soziologisch aufgeklärten Medizinethik“ plädiert vor allem auch für die Rücksichtnahme auf sozialtheoretische Prämissen in sozialempirischen Ansätzen. Tanja Krones widmet sich vor allem der Methodik und Methodologie empirischer Medizinethik in ihrem Aufsatz Empirische Methodologien und Methoden der angewandten und der empirischen Ethik. Ausgehend von der Prämisse, dass der Mensch als bio-psycho-soziales Wesen den wesentlichen Gegenstandsbereich der Medizinethik darstellt, wird der Stellenwert der Sozialwissenschaften als ,empirische Basiswissenschaft“ in der Medizinethik untersucht. Dabei zeichnet sie durchaus ein Spannungsverhältnis zwischen Sozialwissenschaften, allgemeiner und angewandter Ethik nach, das von bestimmten wissenschaftstheoretischen Vorannahmen abhängt.

Im abschließenden Beitrag Empirische Forschung in der Medizinethik: Methodenreflexion und forschungspraktische Herausforderungen am Beispiel eines mixed-method Projekts zur ärztlichen Handlungspraxis am Lebensende diskutieren Jan Schildmann und Jochen Vollmann die Problematik der Integration empirischer und ethischer Aspekte innerhalb eines konkreten Forschungsprojekts. Am Beispiel der Durchführung von quantitativen Umfragen und qualitativen Forschungsinterviews zu klinisch-ethischen Fragestellungen am Lebensende werden methodische und forschungspraktische Herausforderungen interdisziplinärer Forschung in der Medizinethik dargestellt.

Die Herausgeber hoffen, dass dieses Themenheft einen kleinen Beitrag zur Intensivierung der Diskussion über Medizinethik und Empirie in Deutschland leisten kann. Den Bedarf an einer fachinternen Auseinandersetzung und Reflexion über den sogenannten empirical turn 
sehen wir allerdings noch lange nicht gedeckt. Vielmehr wird es in Zukunft darum gehen, eine kleinschrittigere Abstimmung zwischen Ethiktheorie, empirischem Forschungsdesign und theoretischer Interpretation für die Kontextualisierung und Konkretisierung medizinethischer Forschung anzuleiten. Diese und weitere Fragestellungen werden auch im Rahmen der seit 2006 aktiven AEM-Arbeitsgruppe „Ethik und Empirie“ bearbeitet.

Wir möchten uns abschließend bei allen Autorinnen und Autoren für die gute Zusammenarbeit bedanken. Weiterhin danken wir Prof. Dr. Rainer Anselm, Dr. Uwe Fahr, Dr. Markus Rothhaar, Prof. Dr. Christoph Hubenthal, Dr. Rouven Porz und Dr. Micha Werner für die Gutachten im Rahmen des Peer-Review-Verfahrens. Frau Andrea Bojarra, M.A. danken wir herzlichst für die sorgfältige redaktionelle Bearbeitung der Beiträge.

Open Access Dieser Artikel unterliegt den Bedingungen der Creative Commons Attribution Noncommercial License. Dadurch sind die nichtkommerzielle Nutzung, Verteilung und Reproduktion erlaubt, sofern der/die Originalautor/en und die Quelle angegeben sind. 